\title{
Asymptomatic Substernal Goiter Reaching the Diaphragm
}

\author{
${ }^{1}$ Lars J Hansen, ${ }^{2}$ Christoffer H Hahn, ${ }^{3}$ Waldemar Trolle
}

\section{ABSTRACT}

Aim: In addition to presenting a huge substernal goiter (SSG) reaching the diaphragm, we discuss the diagnosis and treatment of SSGs and bilateral benign multinodular goiters.

Background: The presentation of an SSG is usually related to compression of neighboring organs producing symptoms like dyspnoea, dysphonia, and dysphagia. Between 2 to $19 \%$ of all goiters are substernal depending on definition.

Case description: In the present study we discuss a truly unique case of a huge SSG reaching the diaphragm in a 55-year-old woman. Despite the extension of the goiter, the patient was symptom-free. The patient had a history of subtotal thyroidectomy and radioiodine treatment. The SSG was removed surgically and was characterized as a multinodular goiter on pathological examination.

Conclusion: To the best of our knowledge the size, extension, and presentation of the SSG described in this case report is of unprecedented nature.

Clinical significance: There is an ongoing discussion about the management of benign bilateral multinodular goiters. With subtotal resection, the risk of nerve injury and hypoparathyroidism decreases, whereas the risk of recurrent disease increases. The present case is an extreme example of recurrent disease but highly relevant when discussing the management of benign bilateral multinodular goiters.

Keywords: Goiter, Nodular, Substernal.

Search strategy: Literature search was performed on Pubmed. com using the above keywords generated from the Medical Subject Headings (MeSH) Browser on the U.S. National Library of Medicine's homepage.

How to cite this article: Hansen LJ, Hahn $\mathrm{CH}$, Trolle W. Asymptomatic Substernal Goiter Reaching the Diaphragm. World J Endoc Surg 2018;10(3):173-175.

Source of support: Nil

Conflict of interest: None

\footnotetext{
${ }^{1}$ Specialty Registrar, ${ }^{2}$ Consultant and Associate Professor, ${ }^{3}$ Consultant.

1,3 Department of Ear, Nose and Throat, Nordsjællands Hospital, Hillerød, Capital Region, Denmark

${ }^{2}$ Department of Otorhinolaryngology, Head and Neck Surgery and Audiology, Rigshospitalet, Copenhagen, Capital Region, Denmark

Corresponding Author: Lars J Hansen, Specialty Registrar, Department of Ear, Nose and Throat, Nordsjællands Hospital, Hillerød, Capital Region, Denmark, e-mail: larsjuulhansen@ gmail.com
}

\section{BACKGROUND}

Goiter is localized or generalized thyroid hypertrophy. A goiter can be defined as SSG if it extends below the plane of the thoracic inlet or if more than $50 \%$ of its mass is inferior to the thoracic inlet. ${ }^{1}$ Depending on the definition, 2 to $19 \%$ of all goiters are SSGs. ${ }^{1}$ Usually, goiters are divided into primary; originating from aberrant thyroid tissue already present in the mediastinum, or secondary; representing downward growth of cervical thyroid tissue, ${ }^{2}$ the latter accounting for most cases. ${ }^{1}$ The secondary goiters tend to expand into the anterior mediastinum, anterior to the recurrent laryngeal nerve (RLN), whereas $10-15 \%$ descend into the posterior mediastinum.

The majority of SSGs can be managed through a cervical incision. However, in about 2 to $6 \%$ of cases, a sternotomy is required. ${ }^{1,3}$

A recent case series reported that predominantly women suffer from SSGs. ${ }^{4}$ Furthermore, $66 \%$ of the cases presented compression signs, with $46 \%$ having dyspnoea, $26 \%$ dysphonia, and $8 \%$ complaining of dysphagia. ${ }^{4}$

\section{CASE DESCRIPTION}

We present an extraordinary case of a large SSG in a 55-year-old woman. The patient had a history of subtotal thyroidectomy and radioiodine treatment at the age of 18 , with no record of the original pathology. Swelling in the front of the neck had been slowly progressing since then. She recently presented to her general practitioner with cramps in one leg and a low level of thyroid-stimulating hormone (TSH) was found. An endocrinological assessment showed subclinical hyperthyroidism with low TSH and normal thyroxine $\left(\mathrm{T}_{4}\right)$ and triiodothyronine $\left(\mathrm{T}_{3}\right)$ levels. Calcium levels were normal. Scintigraphy showed a large goiter with very low activity levels and evidence of substernal localization (data not shown). The following computed tomography revealed a massive SSG stretching from the neck and to the left diaphragm occupying much of the anterior mediastinum (Fig. 1). The trachea was severely displaced and moderately compressed. Upon examination, a large and firm cervical goiter was found. Fiberoptic laryngoscopy was normal, and the voice was unaffected. Notably, there were no respiratory symptoms or other compression symptoms.

The patient underwent surgery, and under general anesthesia, an extended Kocher's incision joined with 


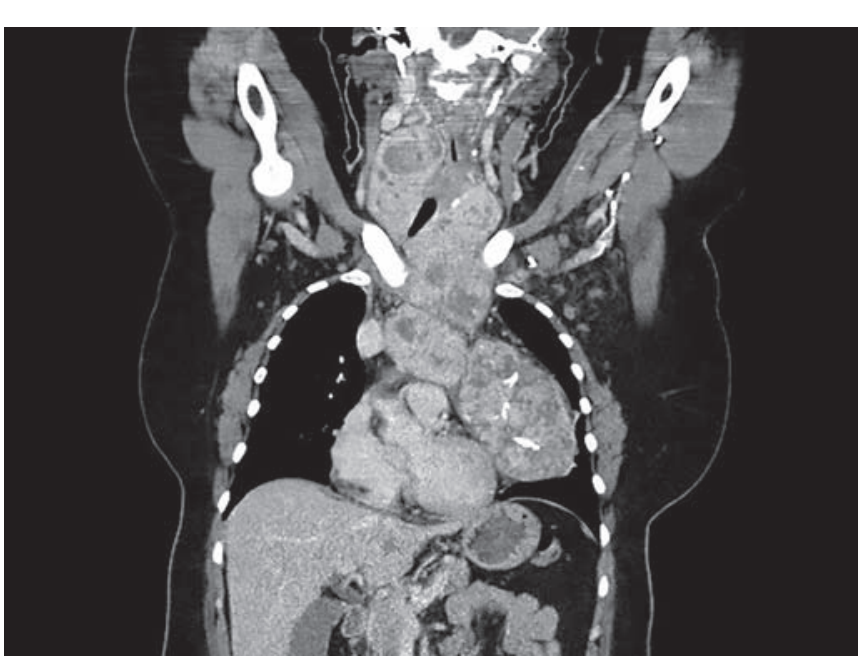

Fig. 1. Coronal computed tomography (CT) scan of the neck and thorax showing the extension of the SSG

a vertical midline incision along the sternum, to allow for a median sternotomy, was made. Under continuous nerve monitoring, the cervical portion of the goiter was removed sparing the RLN on both sides. The cervical incision allowed dissection of the goiter to the level of the aortic arch were the mass was divided. Through a median sternotomy, the remaining SSG was removed from the anterior mediastinum (Fig. 2) sparing the left phrenic nerve. The goiter was adherent to the left pulmonary pleura, part of which had to be resected. The mass had compressed the left lower pulmonary lobe, which was reinflated "peroperatively", as in, during the operation. The goiter was $28 \mathrm{~cm}$ long and had a maximum diameter of $11 \mathrm{~cm}$. The weight was 940 grams (Fig. 3).

Pathological examination showed a bilateral benign multinodular goiter. Postoperative fiberoptic laryngoscopy showed normal movements of the vocal cords. The patient received a short course of antibiotics postoperatively. Low levels of parathyroid hormones (PTH) and calcium were recorded, and the patient was treated

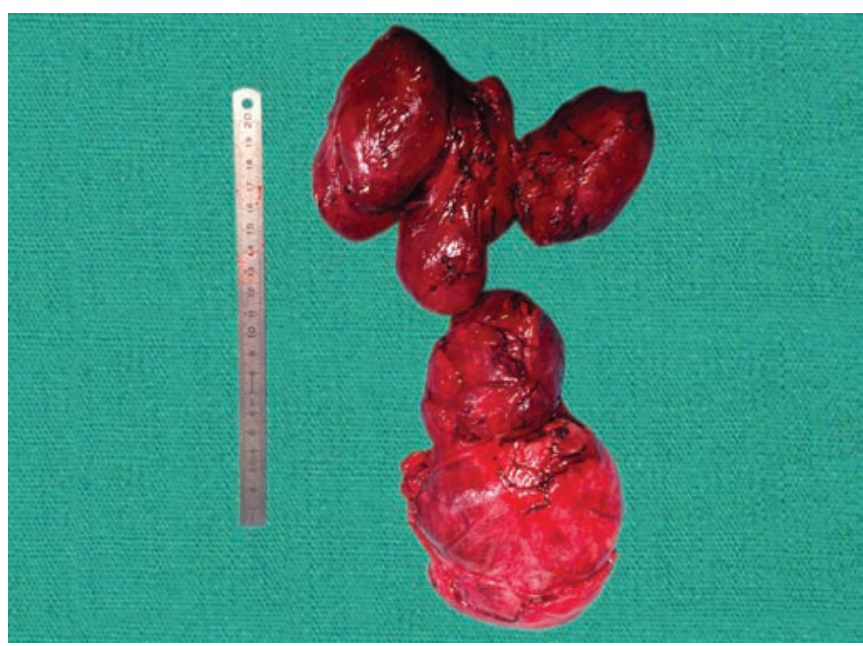

Fig. 3. The removed goiter weighing 940 grams.

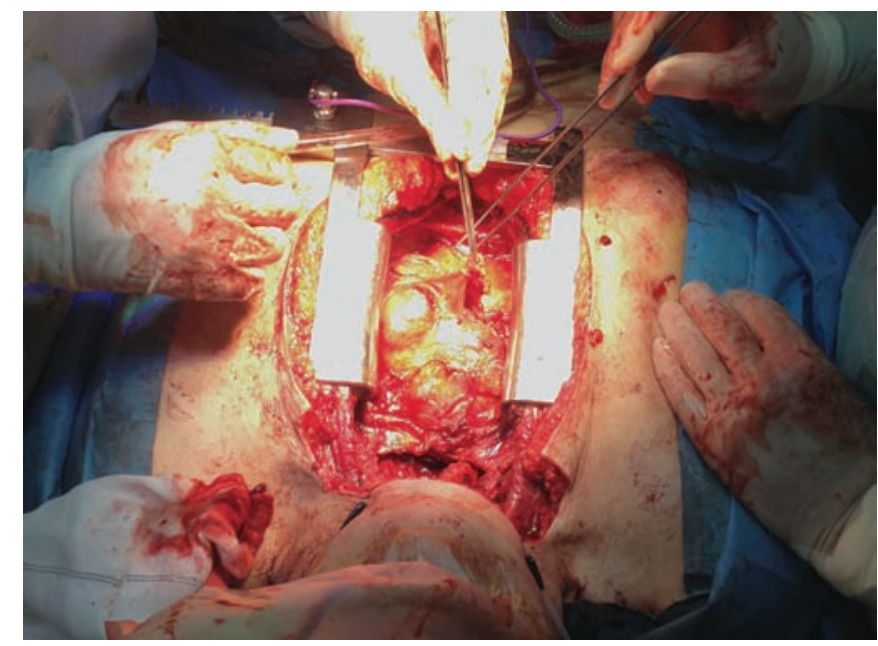

Fig. 2. Median sternotomy revealing the substernal component of the goiter in the anterior mediastinum

with oral calcium supplements. Calcium and PTH levels normalized 2 months after surgery and the patient has otherwise recovered completely.

\section{DISCUSSION}

The size and extension of the SSG in the present study are of unprecedented nature to the best of our knowledge. Besides, it is very rare for an SSG of these proportions to be asymptomatic.

With regards to management, surgery was performed without preceding radioiodine treatment. There is evidence that radioiodine treatment can reduce goiter size by up to $40 \%$, even for SSGs, further augmented by recombinant human $\mathrm{TSH}^{5}{ }^{5}$ However, due to very low activity levels on the preoperative thyroid scintigraphy, radioiodine treatment with or without recombinant TSH would likely have little effect in this case. Also, with increasing compression of lungs and trachea, there was a risk of airway compromise, thus indicating surgical management. Moreover, SSGs have been associated with saddle pulmonary embolisms. ${ }^{6}$

Surgical treatment offers immediate symptom relief. However, there is a higher risk of recurrent laryngeal nerve (RLN) palsy, postoperative hemorrhage, and hypoparathyroidism with surgical removal of an SSG compared to removal of a cervical goiter., ${ }^{7,8}$

Data suggest that 5 to $10 \%$ of operations for SSGs are due to recurrent or persistent goiters, and the most common initial procedures appear to be subtotal thyroidectomy and hemithyroidectomy. ${ }^{1}$ This patient had previously undergone a subtotal thyroidectomy. There is a continuous debate regarding the surgical management of bilateral benign multinodular goiters. With subtotal resection, the incidence of RLN palsy and hypoparathyroidism decreases, on the other hand, the risk of recurrent or persistent disease increases. ${ }^{9}$ The patient in this 
report might be a product of the latter, and therefore worth taking into consideration when discussing surgical management of bilateral benign multinodular goiters.

\section{CONCLUSION}

This case demonstrates that very large SSGs can reach the diaphragm without producing symptoms. The initial thyroid scintigraphy showed some substernal expansion of the mass due to the inactivity of the large intrathoracic component. This stresses the fact, that computed tomography is invaluable in the diagnosis and preoperative assessment of an SSG. Surgical cases like the one described here should be performed electively at a tertiary care center with support from a cardiothoracic team. Finally, the case reminds us that with subtotal thyroidectomy there is a risk of recurrent disease, possibly requiring more extensive surgery.

\section{CLINICAL SIGNIFICANCE}

There is an ongoing discussion about management of benign bilateral multinodular goiters. With subtotal resection, the risk of nerve injury and hypoparathyroidism decreases, whereas the risk of recurrent disease increases. The present case is an extreme example of recurrent disease but highly relevant when discussing the management of benign bilateral multinodular goiters.

In addition, this case demonstrates, that even very large SSGs reaching the diaphragm can be removed surgically as described. This should be done at tertiary care center with support from the cardiothoracic team, and if possible, as an elective case.

\section{ACKNOWLEDGMENT}

Authors would like to thank Elisabeth Arndal, M.D. for the photographic images.

\section{REFERENCES}

1. White ML, Doherty GM, Gauger PG. Evidence-based surgical management of substernal goiter. World J Surg. 2008;32(7):1285-1300.

2. Hashmi SM, Premachandra DJ, Bennett AMD, Parry W. Management of retrosternal goitres: results of early surgical intervention to prevent airway morbidity, and a review of the English literature. J Laryngol Otol 2006;120:644-649.

3. Nankee L, Chen H, Schneider DF, Sippel RS, Elfenbein DM. Substernal goiter: when is a sternotomy required? J Surg Res 2015;199:121-125.

4. Benbakh M, Abou-Elfadl M, Rouadi S, Abada R-L, Roubal M, Mahtar M. Substernal goiter: Experience with 50 cases. Eur Ann Otorhinolaryngol Head Neck Dis 2016;133:19-22.

5. Hegedüs L, Bonnema SJ. Approach to management of the patient with primary or secondary intrathoracic goiter. J Clin Endocrinol Metab 2010;95:5155-5162.

6. Nelson DW, Lopinto M, Sidani C, Lew JI. Large Substernal Thyroid Goiter Associated with Saddle Pulmonary Embolism. WJOES 2016;8:214-216.

7. Testini M, Gurrado A, Avenia N, Bellantone R, Biondi A, Brazzarola $\mathrm{P}$, et al. Does mediastinal extension of the goiter increase morbidity of total thyroidectomy? A multicenter study of 19,662 patients. Ann Surg Oncol 2011;18:2251-2259.

8. Hahn $\mathrm{CH}$, Trolle W, Sørensen $\mathrm{CH}$. Harmonic focus in thyroidectomy for substernal goiter. Auris Nasus Larynx 2015;42:311-317.

9. Mauriello C, Marte G, Canfora A, Napolitano S, Pezolla A, Gambardella $\mathrm{C}$, et al.. Bilateral benign multinodular goiter: What is the adequate surgical therapy? A review of literature. Int J Surg 2016;28 Suppl 1:S7-S12. 